\title{
Propuesta didáctica para la enseñanza de las propiedades de reflexión de las cónicas por medio de la metodología de resolución de problemas ${ }^{1}$
}

\author{
Proposal for teaching the reflective properties of conics \\ through problem solving methodology
}

Proposta de ensino ensinar as propriedades reflexivas de cônicas através da metodologia de resolução de problemacolar"

Recibido: mayo de 2013 Aceptado: agosto de 2013

Angélica Lorena Garzón Muñóz ${ }^{2}$

\section{Resumen}

Al identificar en la enseñanza de la geometría analítica impartida actualmente en Colombia en la educación media en el ciclo V (grados décimo y once) un mayor interés por el desarrollo de cálculos y procesos algebraicos que ofrezcan un resultado numérico por encima de un análisis geométrico; surge el interés por abordar la enseñanza de las propiedades de reflexión de las cónicas por medio del diseño de un propuesta de enseñanza bajo la metodología de resolución de problemas. La propuesta surge a partir de un seguimiento histórico y disciplinar entorno a la temática abordada, donde la resolución de problemas y el modelo de Van Hiele aportan elementos didácticos y epistemológicos para su diseño y aplicación, buscando fortalecer el desarrollo de razonamientos geométricos en los estudiantes.

Palabras clave: Enseñanza; matemáticas escolares; geometría; geometría analítica; propiedades de reflexión de las cónicas; resolución de problemas.

\begin{abstract}
By identifying the teaching of analytic geometry Colombia currently taught in secondary education in the cycle $\mathrm{V}$ (tenth and eleven degrees) greater interest in the development of algebraic calculations and processes that provide a numeric result above geometric analysis; become interested in addressing the teaching of reflective properties of conics through the design of a teaching proposal on the methodology of problem solving. The proposal arises from a historical tracking and discipline around the topics addressed, where the resolution of problems and the Van Hiele model provide didactic and epistemological elements to its design and implementation, seeking to strengthen the development of geometric reasoning in students.
\end{abstract}

1 Artíulo de Investigación

2 Colegio Diego Montaña Cuellar IED, Universidad Nacional De Colombia- Maestria en enseñanza de las ciencias exactas y naturales. Contacto: algarzonm@unal.edu.co 
Keywords: Education; school mathematics, geometry, analytic geometry, reflective properties of conics, resolution of problems.

\section{Resumo}

Ao identificar o ensino da geometria analítica Colômbia atualmente ensinado no ensino secundário no ciclo $\mathrm{V}$ (décimo e onze graus) maior interesse no desenvolvimento de cálculos algébricos e processos que proporcionam um resultado numérico acima análise geométrica; tornar-se interessado em abordar o ensino de propriedades reflexivas de cônicas através da concepção de uma proposta de ensino na metodologia de resolução de problemas. A proposta surge a partir de um rastreamento histórico e disciplina em torno dos temas abordados, onde a resolução de problemas eo modelo de Van Hiele fornecer elementos didáticos e epistemológicos à sua concepção e implementação, buscando fortalecer o desenvolvimento do raciocínio geométrico dos alunos.

Palavras-chave: Educação; matemática escolar, geometria, geometria analítica, as propriedades reflexivas de cônicas, a resolução de problemas.

\section{Presentación del problema}

La enseñanza de la geometría en la educación media se trabaja como un elemento dentro de la Educación matemática, a partir del desarrollo del Pensamiento Espacial y Sistemas Geométricos, uno de los cinco pensamientos que plantean los Lineamientos Curriculares (2006). El objetivo de este pensamiento es desarrollar procesos donde se construya y manipule objetos del plano y el espacio, se creen representaciones mentales de ellos y se reconozcan sus propiedades desde su posición y en relación con los demás. Sin embargo, la educación matemática impartida actualmente como la describe Miguel de Guzmán (2001) trae consigo un abandono de la geometría intuitiva y un incremento por el interés en el desarrollo de destrezas en el cálculo, señalando que: con la sustitución de la geometría por el álgebra la matemática elemental se vació rápidamente de contenidos y de problemas interesantes.

Dentro de la enseñanza de la geometría en general se incluye la geometría analítica, impartida en la educación media específicamente en ciclo V. En esta etapa se enfrenta al estudiante a caracterizar una figura geométrica dentro de un sistema de coordenadas mediante técnicas algebraicas básicas; es decir, se propone realizar un estudio matemático utilizando herramientas de la geometría y el álgebra. Al enseñar cónicas y sus propiedades, se reconoce un fuerte y necesario trabajo en la identificación de las cónicas como curvas, se reconocen los cortes longitudinales de un cono, se reconoce cada una de las ecuaciones que la define, sus gráficas representadas en el plano y en ocasiones se llegan a realizar construcciones geométricas con ellas. Pero el conocimiento de las propiedades geométricas de dichas curvas que nos permiten solucionar situaciones problema interesantes en geometría y algebra, son un elemento que muy pocas veces se llega a tratar perdiendo el carácter aplicativo del objeto matemático estudiado.

Esta problemática motiva el diseño de una propuesta didáctica para estudiantes de ciclo 5 entorno

a la enseñanza de las propiedades de reflexión de las cónicas basada en el planteamiento y resolución de problemas de aplicación que privilegien el análisis geométrico sobre el algebraico.

\section{Marco de referencia conceptual}

El caso particular tratado es el estudio de las cónicas para la que los Estándares Curriculares del 


\section{Ministerio de Educación Nacional (2006)} proponen:

- Identificar en forma visual, gráfica y algebraica algunas propiedades de las curvas que se observan en los bordes obtenidos por cortes longitudinales, diagonales y transversales en un cilindro y en un cono.

- Resolver problemas en los que se usen las propiedades geométricas de figuras cónicas por medio de transformaciones de sus representaciones algebraicas.

- La propuesta diseñada busca fortalecer el trabajo en el aula del segundo estándar estudiando bajo la

- Metodología de resolución de problemas las propiedades de reflexión de las cónicas, que desde Lehman (1989) se reconocen como:

Propiedad de reflexión de la parábola: Si se emiten rayos de luz paralelos al eje de un espejo parabólico, la luz reflejada se concentrará en el foco, así mismo si se emite rayos provenientes del foco hacia el espejo parabólico se reflejaran paralelos al eje del espejo.

Figura 1

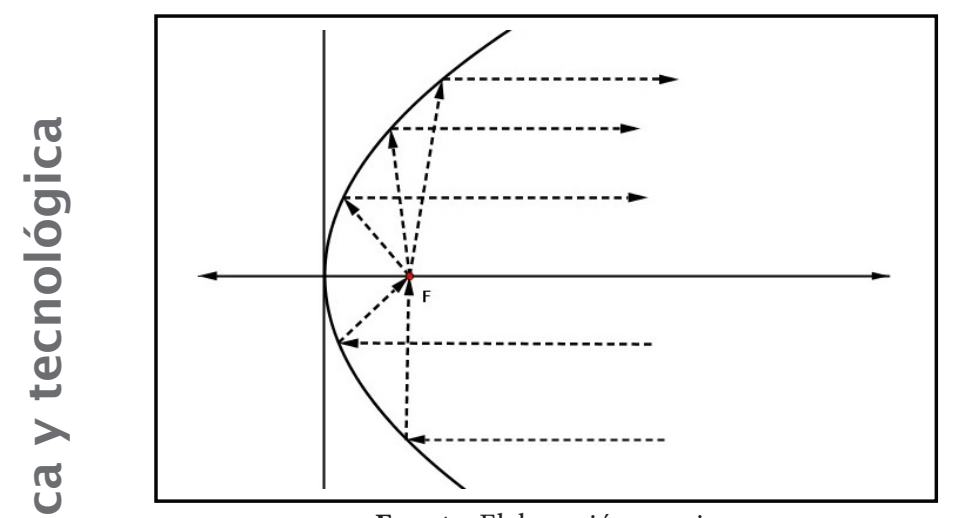

Fuente: Elaboración propia
Figura 2

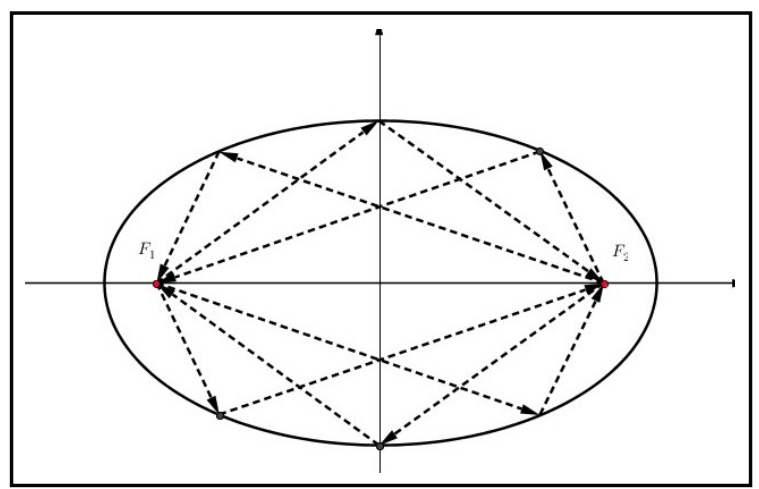

Fuente: Elaboración propia

Propiedad de reflexión de la elipse: Si se emite un Figura 3 rayo de luz desde un foco en el

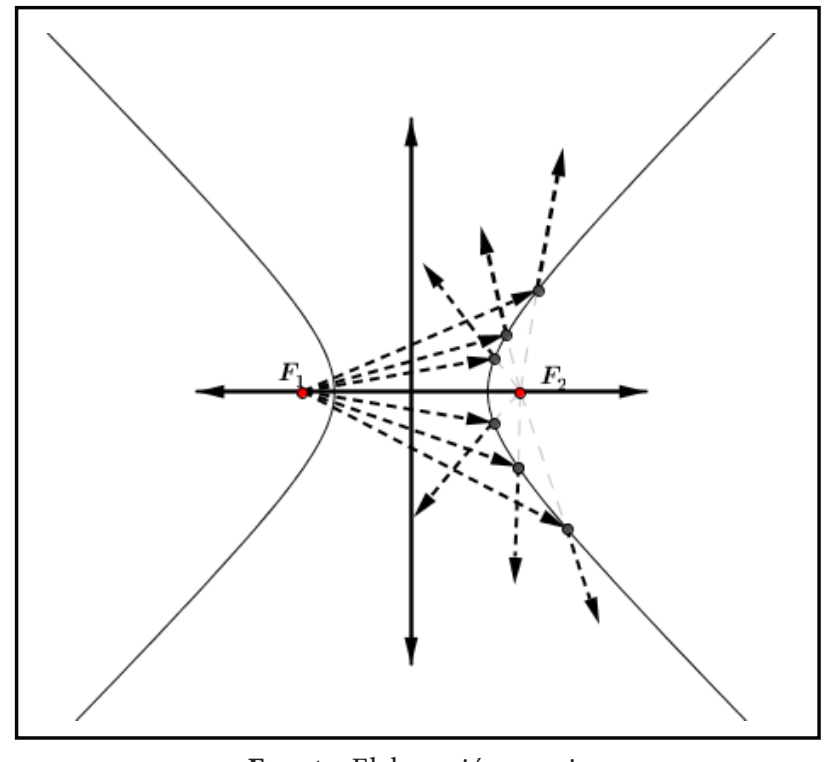

Fuente: Elaboración propia

interior de una elipse, el rayo reflejado se concentrará en el otro foco.

Propiedad de reflexión de la Hipérbola: Si se emite un rayo de luz desde un foco de una rama de una hipérbola hacia la otra rama, el rayo se reflejara de manera que los rayos reflejados parecen provenir del otro foco 
Para el diseño y análisis de la propuesta se hace uso de la caracterización que desarrolla Polya (1989) entorno a la resolución de problemas validando la comprensión de cada una de las situaciones planteadas, y caracterizando las fases de resolución (Comprensión de un problema, concebir un plan, ejecución de un plan, examinar la solución obtenida) en cada caso específico propuesto. Por otra parte el modelo de Van Hiele (1990) modelo educativo de razonamiento y enseñanza de la matemática, principalmente de la geometría plantea una secuencia de tipos de razonamiento de los estudiantes denominados niveles a identificar en el análisis de la propuesta a partir de categorías construidas.

\section{Metodología}

La propuesta didáctica que se plantea se caracteriza por utilizar la metodología de resolución de problemas para el diseño de las actividades y posible aplicación de la propuesta. Se describe esta metodología recurriendo a los Lineamientos curriculares en Matemáticas (2006) y al libro de Polya (1989) titulado "Planteamiento y resolución de problemas".

La resolución y planteamiento de problemas desde los Lineamientos Curriculares es concebida como uno de los cinco procesos generales del currículo en matemáticas ${ }^{3}$; proceso que no tiene un fuerte trabajo en la escuela ya que se evidencia un aprendizaje de las matemáticas formales y abstractas descontextualizadas, donde la resolución de problemas en varias ocasiones se deja al final como parte de la aplicación de la temática trabajada o en ocasiones ni se llega a trabajar.
Miguel de Guzman reconoce la necesidad del uso de situaciones problemáticas exponiendo entre muchas de sus razones para usar esta metodología: la importancia de proporcionar a nuestros jovenes capacidad autónoma para resolver sus propios problemas, y por que muchos de los hábitos que así se consolidan tienen un valor universal, no limitado al mundo de las matemáticas.

\section{Análisis de datos}

Actualmente la propuesta ya fue diseñada bajo un estudio disciplinar y seguimiento

histórico de las cónicas y sus propiedades; y a la fecha se encuentra en etapa de aplicación en el grado décimo jornada mañana del Colegio Diego Montaña Cuellar IED, donde se recopilan datos de la intervención en el aula, notas de campo, entrevistas y soluciones escritas de las situaciones problema. Durante la aplicación haciendo uso de los niveles del modelo de Van Hiele (1990) se crean unas categorías específicas frente a los razonamientos de los estudiantes para enfrentarse al problema, sin embargo a la fecha no se ha realizado un análisis de datos.

\section{Conclusiones}

El trabajo final presentado muestra una propuesta didáctica dirigida a estudiantes de ciclo $\mathrm{V}$

en torno a la enseñanza de las propiedades de reflexión de las cónicas, buscando recuperar de una manera sencilla y atractiva desde el planteamiento de situaciones problema, el desarrollo de análisis geométrico en el aula.

Buscando recuperar el análisis geométrico sobre el algebraico, se reconoce durante la aplicación

3 Los 5 procesos generales del currículo en matemáticas son: Razonamiento, resolución y planteamiento de problemas, la comunicación, la modelación y elaboración, comparación y ejercitación de procedimientos. 
algunos estudiantes que: en búsqueda de una mejor comprensión y generalidad de cada propiedad de reflexión, cuando el estudiante logra relacionar las estrategias que surgen a partir de un análisis geométrico y desarrollar estrategias que fortalecen sus primeros descubrimientos con razonamientos algebraicos, las propiedades de reflexión llegan a concebirse de una manera más formal y global.

Al crear la propuesta bajo la metodología de resolución de problemas se genera un cambio de concepción de la forma y el orden en cómo enseñar cada una de las propiedades de reflexión de las cónicas, ya que, al buscar por medio del planteamiento de una situación la deducción y no la exposición de cada una de las propiedades de reflexión de las cónicas, el orden y la dirección de cómo se dirige un conocimiento (profesor-estudiante) se renueva promoviendo estrategias didácticas en la enseñanza de las propiedades de reflexión de las cónicas.

Ejemplo:

\section{Referencias}

Guzman, M. (2001). Tendencias Actuales de la Educación Matemática. Iralia, 5.

Heath,T. H. (1896). Apollonius of Perga Treatise on conic sections. London: C.J and sons Cambriedge university press warehouse.

Lehmann, C. (1989). Geometría analítica. México: LIMUSA.

Merzbach,U., \& Boyer, C. (2011). A History of Mathematics. Hoboken, New Jersey: Wiley and Sons.

Ministerio de Educación Nacional. (1998). Lineamientos Curriculares de Matemáticas. Serie lineamientos. Áreas obligatorias y Fundamentales. Bogotá: Magisterio.

Ministerio de Educación Nacional. (2006). Estándares Básicos de Competencias en Lenguaje, Matemática, Ciencia y Ciudadanas. Bogotá: Magisterio.

Polya, G. (1989). Como Plantear y Resolver Problemas. Mexico: Trillas.

Rodríguez, A., Pastor, J., \& Gutierrez, A. (1990). Una propuesta de fundamentación para la enseñanza de la geometria: El modelo de Van Hiele. Teoría y Práctica en educación Matemática Alfar, 295-384.
Solo puedes apuntar hacia la tirilla de la lata, No puedes derribar a tus oponentes apuntando directamente a ellos.

Desde la posición que elijas debes derribar a todos tus oponentes sin moverte de tu posición.

Un barco se derriba por completo cuando lo alumbras con el láser perpendicularmente a su base.

Fuente: Elaboración propia 